Pacific Journal of Mathematics

CHARACTERIZATIONS OF COMPLETELY
NONDETERMINISTIC STOCHASTIC PROCESSES 


\title{
CHARACTERIZATIONS OF COMPLETELY NONDETERMINISTIC STOCHASTIC PROCESSES
}

\author{
Peter Bloomfield, Nicholas P. Jewell and Eric Hayashi
}

\begin{abstract}
A discrete weakly stationary Gaussian stochastic process $\{x(t)\}$, is completely nondeterministic if no non-trivial set from the $\sigma$-algebra generated by $\{x(t): t>0\}$ lies in the $\sigma$-algebra generated by $\{x(t): t \leq$ $0\}$. In [8] Levinson and McKean essentially showed that a necessary and sufficient condition for complete nondeterminism is that the spectrum of the process is given by $|h|^{2}$ where $h$ is an outer function in the Hardy space, $H^{2}$, of the unit circle in $\mathrm{C}$ with the property that $h / \bar{h}$ uniquely determines the outer function $h$ up to an arbitrary constant. In this paper we consider several characterizations of complete nondeterminism in terms of the geometry of the unit ball of the Hardy space $H^{1}$ and in terms of Hankel operators.
\end{abstract}

1. Introduction. In [10] Sarason defines a property of a discrete weakly stationary Gaussian stochastic process, $\{x(t)\}$, which he calls complete nondeterminism. This condition is that no set from the future of the process (i.e. the $\sigma$-algebra generated by the random variables $x(t)$ for $t>0$ ) lies in the past (i.e. the $\sigma$-algebra generated by $x(t)$ for $t \leq 0$ ), except for null sets and the complements of null sets. In the spectral representation this condition becomes the following. Let $m$ be the spectral measure of the process and let $\mathcal{P}$ denote the span in $L^{2}(m)$ of the exponentials $e^{i n \theta}$ with $n \leq 0$ where functions are defined on $\mathbf{T}$, the unit circle in $\mathbf{C}$. Let $\mathscr{F}$ denote the span in $L^{2}(m)$ of the exponentials $e^{i n \theta}$ with $n>0$. Then complete nondeterminism is equivalent to the condition that $\mathscr{P} \cap \mathscr{F}=\{0\}$. It is clear that this condition reflects a certain kind of independence (in a statistical sense) of the past, $\mathcal{P}$, and the future, $\mathscr{F}$.

It is of interest to characterize those measures $m$ on $\mathbf{T}$ which lead to completely nondeterministic (cnd) processes. In [10] a necessary and sufficient condition for complete nondeterminism was stated as the measure $m$ being absolutely continuous with respect to Lebesgue measure, $d \theta$, with $\log (d m / d \theta)$ integrable. Unfortunately this characterization is incorrect. In [8, p. 105] Levinson and McKean essentially describe a partial characterization of cnd processes which we discuss in \$3. This paper 
continues an investigation into the problem of characterizing spectral measures of cnd processes.

In $\S 2$ we examine the relationship between complete nondeterminism and some other familiar kinds of independence of $\mathscr{P}$ and $\mathscr{F}$.

In $\$ 3$ we restate the question in several ways which yield answers in terms of exposed points of the unit sphere of $H^{1}$ and certain Hankel operators.

In $\$ 4$ we define $k$-step completely nondeterministic processes and provide the characterization of these processes in terms of cnd processes.

The authors are grateful to D. E. Sarason for some helpful correspondence on the topics of this paper.

2. Complete nondeterminism. A Gaussian process is called deterministic if its past determines the future, i.e., for each $t>0, x(t)$ is measurable with respect to the past. This is translated in the spectral representation to the property that $\mathscr{P}=L^{2}(d m)$. A necessary and sufficient condition for this to occur is that $\log (d m / d \theta)$ be not integrable. Conversely the process is indeterministic if $\log (d m / d \theta)$ is integrable. A stronger restriction than indeterminism is that the process is purely indeterministic or regular. This an asymptotic independence condition which, in the spectral representation, is equivalent to $\cap_{k=1}^{\infty} \mathscr{F}_{k}=\{0\}$ where $\mathscr{F}_{k}$ is the span in $L^{2}(m)$ of the exponentials $e^{i n \theta}$ with $n \geq k$. This condition is often referred to by saying that the process has trivial remote future. Results of Szego [11], Kolmogorov [6] and Krein [7] show that $\{x(t)\}$ is regular if and only if $m$ is absolutely continuous with respect to Lebesgue measure and $\log (d m / d \theta)$ is integrable. First we give an example of a process which is regular but not completely nondeterministic, thereby showing that the characterization in [10] is incorrect. We will use the following notation. $L^{1}$ (resp. $L^{2}$ ) is the space of integrable (resp. square integrable) functions on T. $L^{\infty}$ is the space of essentially bounded functions on $\mathbf{T}$. We shall often regard functions in $L^{1}$ as extended harmonically into the open unit disc $D=\{z:|z|<1\}$ by means of Poisson's formula. We let $H^{1}$ denote those functions in $L^{1}$ which have analytic extensions into the disc. We define $H^{2}$ and $H^{\infty}$ similarly. $H^{2}$ is a Hilbert space with orthonormal basis $\left\{z^{n}: n=0,1,2, \ldots\right\}$. For standard results on the Hardy spaces we refer to [5].

For a regular process we can write $d m=w d \theta=|H| d \theta=|h|^{2} d \theta$ where $H$ is an outer function in $H^{1}$ and $h$ is an outer function in $H^{2} . w$ is known as the spectral density of the process. 
PROPOSITION 1. There is a regular process which is not completely nondeterministic.

Proof. Let $w\left(e^{i \theta}\right)=\left|1+e^{i \theta}\right|^{2}=|1+z|^{2}$ and put $d m=w d \theta$. Since $\log |1+z|^{2} \in L^{1}$ this process is regular. However $(1+\bar{z})^{-1} \in \mathcal{P} \cap \mathcal{F}$. This follows since $1+z$ is outer. For we have

$$
\lim _{n \rightarrow \infty} \int_{\mathbf{T}}\left|1-p_{n}(1+z)\right|^{2} d z=0
$$

for some sequence $p_{n}$ of polynomials in $z$;

hence

$$
\begin{aligned}
\int_{\mathbf{T}}\left|(1+\bar{z})^{-1}-z p_{n}\right|^{2}|1+\bar{z}|^{2} d z \rightarrow 0 \quad \text { as } n & \rightarrow \infty \\
\Rightarrow \int_{\mathbf{T}}\left|(1+\bar{z})^{-1}-z p_{n}\right|^{2}|1+z|^{2} d z & \rightarrow 0 \quad \text { as } n \rightarrow \infty ;
\end{aligned}
$$

i.e. $(1+\bar{z})^{-1} \in \mathscr{F}$. Similarly

$$
\begin{aligned}
\int_{\mathbf{T}}\left|(1+\bar{z})^{-1}-\bar{p}_{n}\right|^{2}|1+z|^{2} d z & \\
& =\int_{\mathbf{T}}\left|1-p_{n}(1+z)\right|^{2} d z \rightarrow 0 \text { as } n \rightarrow \infty ;
\end{aligned}
$$

i.e. $(1+\bar{z})^{-1} \in \mathcal{P}$.

We next obtain a simple necessary and sufficient condition for complete nondeterminism. It is straightforward to see that if $m$ is singular with respect to Lebesgue measure then $\mathscr{P} \cap \mathscr{F} \neq\{0\}$. This, together with earlier comments means that in considering cnd processes we can restrict our attention to regular processes.

We wish to rephrase our question in terms of $L^{2}$ rather than $L^{2}(m)$. We have $d m=|h|^{2} d \theta$. Consider the mapping $T: L^{2}(m) \rightarrow L^{2}$ given by $T f=h f$. It is easily verified that $T$ is an isometry of $L^{2}(m)$ onto $L^{2}$. Also $T$ maps $\mathscr{F}$ onto $H_{0}^{2}=\left\{f \in H^{2}: f(0)=0\right\}$, and $T$ maps $\mathcal{P}$ onto $(h / \bar{h}) \overline{H^{2}}$ where $\overline{H^{2}}=\left\{\bar{f}: f \in H^{2}\right\}$.

Proposition 2. A process is not cnd if and only if $h / \bar{h}=\alpha(F / \bar{F})$ where $F \in H^{2}$ is outer and $\alpha$ is inner with $\alpha(0)=0$. 
Proof. Using the isometry $T$ we see that $\mathscr{P} \cap \mathscr{F} \neq\{0\}$ if and only if there are non-zero functions $g_{1}, g_{2}$ in $H^{2}$ such that

$$
\begin{aligned}
z g_{1} & =(h / \bar{h}) \bar{g}_{2} \\
& \Leftrightarrow z\left(g_{1} / h\right)=\left(\bar{g}_{2} / \bar{h}\right) \text { and } z\left(g_{2} / h\right)=\left(\bar{g}_{1} / \bar{h}\right) \\
& \Rightarrow z\left(g_{1}+g_{2}\right) / h=\left(\overline{g_{1}+g_{2}}\right) / \bar{h} .
\end{aligned}
$$

Hence $\mathscr{P} \cap \mathscr{F} \neq\{0\}$ if and only if there exists a function $G \in H^{2}$ such that $z G / h=\bar{G} / \bar{h}$. If we use the inner-outer factorization of $G$ then this equality becomes

$$
\begin{aligned}
z \phi F / h & =\overline{\phi F} / \bar{h} \quad \text { where } \phi \text { is inner and } F \in H^{2} \text { is outer, } \\
& \Rightarrow h / \bar{h}=\alpha(F / \bar{F}) \text { and } \alpha(0)=0 .
\end{aligned}
$$

Conversely

$$
\begin{aligned}
h / \bar{h} & =\alpha(F / \bar{F}), \quad \alpha(0)=0 \\
& \Rightarrow(h / \bar{h}) \bar{F}=z(\beta F) \quad \text { where } \alpha=z \beta \\
& \Rightarrow \mathscr{P} \cap \mathcal{F} \neq\{0\} \quad \text { by the above. }
\end{aligned}
$$

The same reasoning yields the following result for $k \geq 1$ :

$\mathscr{P} \cap \mathscr{F}_{k} \neq\{0\} \Leftrightarrow h / \bar{h}=\alpha(F / \bar{F}) \quad$ where $F \in H^{2}$ is outer and $\alpha$ is inner with $\alpha$ having a zero at the origin of order at least $k$.

Another strictly stronger property than regularity is that of minimality. Introduced by Kolmogorov [6] this property says that a process is minimal if the value of the random variable $x(0)$ cannot be predicted without error from the values of the random variables $\{x(t): t \neq 0\}$. In other words a process is not minimal if it is possible to perfectly interpolate any value of the process from knowledge of the remaining values of the process. Kolmogorov [6] proved that a process is minimal if and only if $w^{-1}$ is in $L^{1}$.

It is immediately of interest to examine the relationship between minimal processes and completely nondeterministic processes.

Proposition 3. If the process $\{x(t)\}$ is minimal then it is completely nondeterministic. On the other hand there exist completely nondeterministic processes which are not minimal.

Proof. Suppose $\{x(t)\}$ is minimal. Then by Kolmogorov's theorem $h^{-1} \in H^{2}$. Using Proposition 2 we argue by contradiction. For suppose 
$\{x(t)\}$ is not completely nondeterministic. Then $(h / \bar{h})=\alpha(f / \bar{f})$ where $f$ is outer and $\alpha$ is inner with $\alpha(0)=0$. This equality implies $\bar{f} / \bar{h}=\alpha(f / h)$. The LHS is in $\overline{H^{1}}$ and the RHS is in $H_{0}^{1}$ which forces both sides to be zero and thus $f=0$ which is a contradiction. This proves the first statement of the proposition. An example of a process which yields the second statement is given by $w=|1+z|$. In this case $h=(1+z)^{1 / 2}$ and $h / \bar{h}=z^{1 / 2}$. By Kolmogorov's criterion this process is not minimal. On the other hand suppose $h / \bar{h}=\alpha(f / \bar{f})$ for $f$ outer, $\alpha$ inner with $\alpha(0)=0$. Then

$$
\begin{aligned}
z^{1 / 2} & =\alpha(f / \bar{f})=z \phi(f / \bar{f}) \quad \text { with } \phi \text { inner } \\
& \Rightarrow z^{1 / 2} \phi f=\bar{f} \Rightarrow z(\phi f)^{2}=(\bar{f})^{2} .
\end{aligned}
$$

The LHS is in $H_{0}^{1}$ and the RHS is in $\overline{H^{1}}$. Again this forces both sides to be zero and hence $f=0$ which gives a contradiction. Thus the process with $w=|1+z|$ is completely nondeterministic.

Let $\mathscr{P}_{k}$ be the span in $L^{2}(m)$ of the exponentials $e^{i n \theta}$ with $n \leq k$. A minimal process is one for which the function 1 does not belong to the closed linear span of $\mathscr{P}_{1}$ and $\mathscr{F}_{1}$ i.e. $1 \notin \mathscr{P}_{1} \vee \mathscr{F}_{1}$. There is a similar restatement of the condition of completely nondeterminacy. Let $\mathscr{P}_{1}+\mathscr{F}_{1}$ $=\left\{f \in L^{2}(m): f=g+h\right.$ with $\left.g \in \mathscr{P}_{1}, h \in \mathscr{F}_{1}\right\}$.

Proposition 4. A regular process is completely nondeterministic if and only if $1 \notin \mathscr{P}_{1}+\mathscr{F}_{1}$.

Proof. Assume $f$ is a non-zero element of $\mathscr{P} \cap \mathcal{F}$. Then, for some $k \geq 1, f \in \mathscr{F}_{k}$ but $f \notin \mathscr{F}_{k+1}$ (since $\cap_{k=1}^{\infty} \mathscr{F}_{k}=\{0\}$ ). Hence $f=a e^{i k \theta}+f_{1}$ where $a \neq 0$ and $f_{1} \in \mathscr{F}_{k+1}$. This implies

$$
\begin{aligned}
e^{i k \theta} & =\left(f-f_{1}\right) / a \in \mathscr{P}+\mathscr{F}_{k+1} \\
& \Rightarrow 1 \in\left(e^{-i k \theta \mathscr{P}}\right)+\mathscr{F}_{1} \subseteq \mathscr{P}_{1}+\mathscr{F}_{1} .
\end{aligned}
$$

Conversely assume that $1 \in \mathscr{P}_{1}+\mathscr{F}_{1}$. Then $1=f_{1}+f_{2}$ with $f_{1} \in \mathscr{P}_{1}, f_{2} \in$ $\mathscr{F}$. Hence $e^{i \theta} f_{1}=e^{i \theta}-e^{i \theta} f_{2} \in \mathscr{F}$. But $e^{i \theta} f_{1} \in \mathscr{P}$. Hence $e^{i \theta} f_{1} \in \mathscr{P} \cap \mathscr{F}$.

3. Exposed points of the ball in $H^{1}$ and Hankel operators. It is well known (see [2]) that the extreme points of the unit ball of $H^{1}$ are given by the outer functions $F$ in $H^{1}$ with $\|F\|_{1}=1$. It is also well known that an $H^{1}$ function $F$ of unit norm is not determined by its argument.

In [8, p. 205] Levinson and McKean showed that for continuous processes the dimension of $\mathscr{P} \cap \mathscr{F}_{0}=1$ if and only if $h / \bar{h}$ determines the outer function $h$ up to a constant. In this section we consider this 
approach which is closely related to the results of $\$ 2$ and consider this characterization in geometrical terms.

In their study of extremum problems in $H^{1}$ deLeeuw and Rudin introduced the following sets of $H^{1}$ functions indexed by unimodular $L^{\infty}$ functions. Let $\phi \in L^{\infty}$ with $|\phi|=1$ almost everywhere and define

$$
S_{\phi}=\left\{F \in H^{1}:\|F\|_{1}=1, F /|F|=\phi \text { almost everywhere }\right\} \text {. }
$$

Geometrically $S_{\phi}$ is the intersection of the ball of $H^{1}$ and the hyperplane $\left\{F \in H^{1}: \int \phi F d \theta=1\right\}$ and so $S_{\phi}$ is a convex set (which may be empty, in general). When $S_{\phi}$ contains exactly one function $F$, the hyperplane touches the ball of $H^{1}$ only at $F$ which means that $F$ is an exposed point of the ball of $H^{1}$. (In fact the definition of $S_{\phi}$ we have given corresponds to $S_{\phi}^{-}$as defined as deLeeuw and Rudin.)

Proposition 5. Let $w=|H|=|h|^{2}$. Without loss of generality assume that $\int w d \theta=1$. The following statements are equivalent:

(1) $\{x(t)\}$ is completely nondeterministic,

(2) $S_{h / \bar{h}}$ contains exactly one function,

(3) $h^{2}=H$ is an exposed point of the unit ball in $H^{1}$.

Proof. Note that $S_{h / h}{ }^{-}$always contains $h^{2}$, so that our comments above show the equivalence of (2) and (3). Now suppose that $\{x(t)\}$ is not completely nondeterministic. By Proposition $2, h / \bar{h}=\alpha(F / \bar{F})$ where $\alpha$ is inner and $\alpha(0)=0$ and $F \in H^{2}$ is outer. Hence $\alpha F^{2} /\left|\alpha F^{2}\right|=\alpha F^{2} /|F|^{2}$ $=\alpha F / \bar{F}=h / \bar{h}$. Thus a positive multiple of $\alpha F^{2}$ is in $S_{h / \bar{h}}$. But $a\left(\alpha F^{2}\right)$ $\neq h^{2}$ for any $a>0$ since $\alpha$ has a zero at the origin. Hence $S_{h / h}$ contains more than one function. Conversely suppose $S_{h / h}$ contains more than one function. Then, by Theorem 9 of [2] $S_{h / \bar{h}}$ contains a function $f$ with $f(0)=0$. Write $f=b F^{2}$ where $b$ is inner, $b(0)=0$, and $F \in H^{2}$ is outer. Now $f \in S_{h / h}$ implies that $h / \bar{h}=b F / \bar{F}$ which, by Proposition 2, shows that $\{x(t)\}$ is not completely nondeterministic.

A similar result is given in the following proposition for $k \geq 1$.

Proposition 6. $\mathscr{P} \cap \mathscr{F}_{k} \neq\{0\}$ if and only if there is a function $f \in S_{h / h}$ where $f$ has $k$ zeros (counting multiplicities) in the open unit disc.

Proof. By Proposition 2, $\mathscr{P} \cap \mathscr{F}_{k} \neq\{0\}$ implies that $h / \bar{h}=z^{k} \phi(F / \bar{F})$ where $\phi$ is inner and $F \in H^{2}$ is outer. As in the proof of Proposition 5 it 
follows that $z^{k} \phi F^{2} \in S_{h / \bar{h}}$. Conversely if $f \in S_{h / \bar{h}}$ and $f\left(z_{1}\right)=f\left(z_{2}\right)=$ $\cdots=f\left(z_{k}\right)=0$ where $z_{j} \in D(1 \leq j \leq k)$ then it is easy to verify that a positive multiple of

$$
g(z)=z^{k} f(z) \prod_{j=1}^{k}\left(z-z_{j}\right)^{-1}\left(1-\bar{z}_{j} z\right)^{-1}
$$

is in $S_{h / \bar{h}}$. Factorize $g$ as $g=z^{k} b F$ where $b$ is inner and $F \in H^{2}$ is outer. Since $a g \in S_{h / h}$ for some $a>0$ it follows that $h / \bar{h}=z^{k} b F / \bar{F}$ showing that $\mathscr{P} \cap \mathscr{F}_{k} \neq\{0\}$.

Note that Proposition 5 yields the version of the Levinson and McKean result as applied to cnd processes: namely, a process is cnd if and only if $\arg (h / \bar{h})$ is the argument of a unique $H^{1}$ function.

Since we have expressed the characterization of completely nondeterministic processes in terms of an extremum problem it is not suprising that there is a version of the problem in terms of the norms of Hankel operators, which are closely related to extremum problems on $H^{1}$.

Let $P$ be the orthogonal projection of $L^{2}$ onto $H^{2}$. Recall that the Hankel operator with symbol $\phi \in L^{\infty}$ is the bounded operator from $H^{2}$ to $L^{2} \ominus H^{2}$ defined by

$$
H_{\phi}(f)=(I-P)(\phi f) \quad\left(f \in H^{2}\right) .
$$

The norm of $H_{\phi}$ is given by $\left\|H_{\phi}\right\|=d\left(\phi, H^{\infty}\right)=\inf _{f \in H^{\infty}}\|\phi-f\|_{\infty}$. It is straightforward to show from first principles that the process $\{x(t)\}$ is not cnd if and only if $H_{h / h}^{-}$attains the norm of 1 on the unit sphere of $H^{2}$. In fact more is true.

In [1] it is essentially shown that $H_{\phi}$ attains its norm on the unit sphere on $H^{2}$ if and only if $\phi=f+\lambda \psi$ where $f \in H^{\infty}, \lambda>0$ and $|\psi|=1$ a.e. on $\mathbf{T}$ with $S_{\psi}^{-}$containing more than one function. Also if $\|\phi\|_{\infty}=1$ then $H_{\phi}$ attains the norm 1 if and only if $|\phi|=1$ a.e. on $\mathbf{T}$ and $S_{\psi}^{-}$contains more than one function [1]. There is another result of this type which does not seem to have appeared in the literature.

Proposition 7. $\left\|H_{\phi}\right\|<\|\phi\|_{\infty} \Rightarrow \phi=f+\lambda \psi$ where $f \in H^{\infty}, \lambda>0$ and $|\psi|=1$ a.e. on $\mathbf{T}$ with $S_{\psi}^{-}$containing exactly one function.

Proof. Without loss of generality we assume that $\|\phi\|_{\infty}=1$. Suppose $\left\|H_{\phi}\right\|<1$. Then by [3] there exists $\psi \in L^{\infty}$ such that (i) $\phi-\psi \in H^{\infty}$ and (ii) $\psi=\bar{F} /|F|$ for some $F \in H^{1}, F \neq 0$. Now (i) $\Rightarrow H_{\psi}=H_{\phi}$ and so $\left\|H_{\psi}\right\|<1$. So there exists $g \in H^{\infty}$ such that $\|(\bar{F} /|F|)-g\|_{\infty}=a<1$ which gives that $|\arg (g F)|<b<\pi / 2$. Hence $(g F)^{-1} \in H^{1}$ (since $g F \neq 0$ 
on $D$ and if $G$ is analytic on $D$ and $|\arg G|<b<\pi / 2$ then $G \in H^{p}$ for all $p<\pi / 2 b)$. Thus $g(g F)^{-1} \in H^{1} \Rightarrow F^{-1} \in H^{1}$. Now $F /|F|=\bar{\psi}$ so that a positive multiple of $F$ is in $S_{\psi}^{-}$. Then $F^{-1} \in H^{1}$ implies that $S_{\psi}^{-}$contains one and only one function (if $G \in S_{\beta}$ and $G^{-1} \in H^{1}$ then $S_{\beta}=\{G\}$-see [2, Theorem 8] and use the fact that positive $H^{1 / 2}$ functions are constant).

Note however that $S_{\psi}^{-}$containing exactly one function does not necessarily imply that $\left\|H_{\psi}\right\|<\|\psi\|_{\infty}$. For example if $h=(1+z)^{1 / 2}$, and we take $\psi=\bar{h} / h$ it can be shown that $\left\|H_{\psi}\right\|=1$ but, as we saw in the proof of Proposition 3, $|h|^{2}$ corresponds to a cnd process so that $S_{h / h}=$ $\left\{h^{2}\right\}$.

4. $k$-Step completely nondeterministic processes. The Helson-Szego theorem gives an elegant characterization of the spectral densities of processes where $\mathscr{P}$ and $\mathscr{F}_{1}$ are at positive angle i.e. $\sup \{|(f, g)|: f \in$ unit ball of $\mathscr{P}, g \in$ unit ball of $\mathscr{F}\}<1$. See [10] for a discussion of this result. Note that such processes are necessarily cnd. In the course of characterizing processes with $\mathcal{P}$ and $\mathscr{F}_{k}$ at positive angle Helson and Sarason [4] showed that the spectral density of such a process, $w$, is given by $w=|p|^{2} w_{1}$ where $w_{1}$ is the spectral density of a process with $\mathscr{P}$ and $\mathscr{F}_{1}$ at positive angle and $p$ is a polynomial of degree less than $k$ with zeros all on T.

In this section we show that there is a similar theory relating processes with $\mathscr{P} \cap \mathscr{F}_{k}=\{0\}$ and cnd processes. We call a process $k$-step completely nondeterministic $\left(k\right.$-cnd) if $\mathscr{P} \cap \mathscr{F}_{k}=\{0\}$ but $\mathscr{P} \cap \mathscr{F}_{k-1} \neq\{0\} \quad(k \geq 1)$. For simplicity we let $M_{k}$ denote the image of $P \cap \mathscr{F}_{k}$ in $L^{2}$ under the isometry $T$. Thus $M_{k}=\{0\}$ if and only if $\mathscr{P} \cap \mathscr{F}_{k}=\{0\}$.

Proposition 8. Suppose that $w=|p|^{2} w_{0}$, where $p$ is a polynomial of degree $k$ with all $k$ zeros on $\mathbf{T}$, and $w_{0} \in L^{1}$. Then $\mathscr{P} \cap \mathscr{F}_{k} \neq\{0\}$.

Proof. We show that $1 / \bar{p} \in \mathcal{P} \cap \mathscr{F}_{k}$.

(i) $1 / \bar{p} \in \mathcal{P}$ : without loss of generality we can assume that

$$
\begin{aligned}
1 / \bar{p} & =\prod_{j=1}^{n}\left(1-\bar{z} / \bar{\zeta}_{j}\right)^{-n_{j}}, \quad\left|\zeta_{j}\right|=1 \\
& =\sum_{j=1}^{n} q_{n_{j}-1}(\bar{z})\left(1-\bar{z} / \bar{\zeta}_{j}\right)^{-n_{j}} \quad \text { where } q_{n_{j}-1} \text { is a polynomial. }
\end{aligned}
$$


Now $\left(1-\bar{z} / \bar{\zeta}_{j}\right)^{-n_{J}}$ can be approximated by polynomials in $\bar{z}$ in $L^{2}(m)$. In fact

$$
\begin{gathered}
\int_{\mathbf{T}} \mid\left(1-\bar{z} / \bar{\zeta}_{j}\right)^{-n_{J}}-\left\{1+\frac{n-1}{n}\left(\bar{z} / \bar{\zeta}_{j}\right)+\frac{n-2}{n}\left(\bar{z} / \bar{\zeta}_{J}\right)^{2}\right. \\
\left.+\cdots+(1 / n)\left(\bar{z} / \bar{\zeta}_{j}\right)^{n-1}\right\}\left.^{n_{j}}\right|^{2} w(\theta) d \theta \\
=\int_{\mathbf{T}} \mid 1-\left[( 1 - \overline { z } / \overline { \zeta } _ { j } ) \left\{1+\frac{n-1}{n}\left(\bar{z} / \bar{\zeta}_{j}\right)\right.\right. \\
\left.\left.+\cdots+(1 / n)\left(\bar{z} / \bar{\zeta}_{j}\right)^{n-1}\right\}\right]\left.^{n_{j}}\right|^{2} w_{2}(\theta) d \theta \\
\text { where } w_{2}=w /\left|1-z / \zeta_{j}\right|^{n_{J}} \\
=\int_{\mathbf{T}}\left|1-\left[1-(1 / n)\left\{\bar{z} / \bar{\zeta}_{j}+\cdots+\left(\bar{z} / \bar{\zeta}_{j}\right)^{n}\right\}\right]^{n_{J}}\right|^{2} w_{2}(\theta) d \theta \\
\rightarrow 0 \text { as } n \rightarrow \infty \text { by Lebesgue's dominated convergence theorem. }
\end{gathered}
$$

Hence $1 / \bar{p} \in \mathcal{P}$.

(ii) $1 / \bar{p} \in \mathscr{F}_{k}: 1 / \bar{p}=z^{k} / z^{k} \bar{p}=z^{k} / q_{k}$ where $q_{k}=z^{k} \bar{p}$ is also a polynomial of degree $k$ in $z$. The same construction as in (i) shows that $1 / q_{k}$ can be approximated by polynomials in $z$ in $L^{2}(m)$. Hence $1 / q_{k} \in \mathscr{F}_{0}$ and $1 / \bar{p} \in \mathscr{F}_{k}$.

REMARK. This proposition implies that if we restrict our attention to cnd processes then the strong mixing condition implies the property that $\mathscr{P}$ and $\mathscr{F}_{1}$ be at positive angle; (see [4], [10, p. 77] for definitions). For if the angle between $\mathscr{P}$ and $\mathscr{F}_{n}$ is converging to $\pi / 2$ as $n \rightarrow \infty$ then, for some $k, \mathscr{P}$ and $\mathscr{F}_{k}$ are at a positive angle which implies by [4] that $w=|p|^{2} w_{1}$ for some trigonometric polynomial $p$ where $w_{1}$ is the spectrum of a process for which $\mathscr{P}$ and $\mathscr{F}_{1}$ are at a positive angle. If the process is cnd then Proposition 5 implies that $p$ must have zero degree. In general the strong mixing condition does not imply that $\mathscr{P}$ and $\mathscr{F}_{1}$ are at positive angle (e.g. take $h=1+z$ ).

Proposition 9. Let $k \geq 1$. Suppose $M_{k}=\{0\}$ but $M_{k-1} \neq\{0\}$. Then $h / \bar{h}=z^{k-1} F / \bar{F}$ where $F$ is outer and $|F|^{2}$ corresponds to $a$ cnd process. 
Proof. $\mathscr{P} \cap \mathscr{F}_{k-1} \neq\{0\}$ implies that $h / \bar{h}=z^{k-1} \alpha(F / \bar{F})$ where $F \in$ $H^{2}$ is outer, $\alpha$ is inner. Suppose that $\alpha$ is non-constant. Then we can find constants $a, b$ such that $0 \neq a F+b(\alpha F) \in H_{0}^{2}$. Then

$$
z^{k-1}(a F+b \alpha F) / h=\overline{(\bar{a} \alpha F+\bar{b} F)} / \bar{h} .
$$

Then LHS $\in \mathscr{F}_{k}$ and the RHS $\in \mathscr{P}$. Hence $\mathscr{P} \cap \mathscr{F}_{k} \neq\{0\}$. This contradiction implies that $\alpha$ is a constant $\lambda$, which can then be absorbed into $F$. $M_{k}=\{0\}$ implies that $F$ must correspond to a cnd process.

Proposition 10. Let $k \geq 0$. If $M_{k}=M_{k+1}$ then $M_{k}=\{0\}$.

Proof. Observe that $M_{k}=\left[(h / \bar{h}) H_{0}^{2}+z^{k-1} \overline{H^{2}}\right]^{\perp}$. Thus

$$
M_{k}=M_{k+1} \Rightarrow z^{k} \in(h / \bar{h}) H_{0}^{2}+z^{k-1} \overline{H^{2}} .
$$

This gives

$$
z^{k+1} \in(h / \bar{h}) z H_{0}^{2}+z^{k} \overline{H^{2}} \subseteq(h / \bar{h}) H_{0}^{2}+z^{k-1} \overline{H^{2}} \quad \text { by }(*) .
$$

Similarly $z^{k+2}, z^{k+3}, \ldots$ belong to $(h / \bar{h}) H_{0}^{2}+z^{k-1} \overline{H^{2}}$. But this implies that $L^{2}=\bigvee_{j=1}^{\infty} z^{k+j} H^{2} \subseteq M_{k}^{\perp}$. Hence $M_{k}=\{0\}$.

THEOREM 11. The spectral density $w$ is the spectral density of a $k$-cnd process if and only if $w=|p|^{2} w_{0}$ where $p$ is a polynomial of degree $k-1$ with all of its zeros on $\mathbf{T}$ and $w_{0}$ is the spectral density of $a$ cnd process.

Proof. Suppose $w=|h|^{2}$ corresponds to a $k$-cnd process. Since $\operatorname{dim}\left(M_{j+1}^{\perp} / M_{j}^{\perp}\right) \leq 1$, it follows from Proposition 10 that $\operatorname{dim}\left(M_{j-1} / M_{j}\right)$ $=1$ for $j=1, \ldots, k$. Thus $\operatorname{dim} M_{0}=k$. Now $M_{0}=(h / \bar{h}) \overline{H^{2}} \cap H^{2}=$ $\left[(h / \bar{h}) H_{0}^{2}+\bar{z} \bar{H}^{2}\right]^{\perp}$. Also Proposition 9 shows that $h / \bar{h}=z^{k-1} F / \bar{F}$ where $w_{0}=|F|^{2}$ is the spectral density of a cnd process. It is easy to check that $z^{J} F \in M_{0}$ for $j=0, \ldots, k-1$. Hence $M_{0}=\bigvee\left\{F, z F, \ldots, z^{k-1} F\right\}$. But $h \in M_{0}$ and so $h=p F$ where $p$ is a polynomial of degree $k-1$. It is clear that since $|h|^{2}$ is $k$-cnd $p$ must have exactly $k-1$ roots on $\mathbf{T}$ counting multiplicities. Conversely, if $w=|p|^{2} w_{0}$ where $p, w_{0}$ are as in the theorem, then Proposition 8 shows that $\mathscr{P} \cap \mathscr{F}_{k-1} \neq\{0\}$. The fact that $w_{0}$ corresponds to a cnd process gives $\mathscr{P} \cap \mathscr{F}_{k}=\{0\}$.

REMARK. There are some intriguing questions concerning $S_{h / h}$ which are related to the work of this Section. In [2] it was shown that $S_{h / h}=\left\{h^{2}\right\}$ implies that $h$ is strong outer, i.e. $h /(z-\lambda) \notin H^{2}$ for all $\lambda \in \mathbf{T}$. It would be of interest to construct a strong outer function $h$ with $S_{h / h} \neq\left\{h^{2}\right\}$. In such a case $\mathscr{P} \cap \mathscr{F}_{k}$ is infinite dimensional for all $k \geq 1$. This follows since if $0 \neq \operatorname{dim} M_{1}=n<\infty$ then, by the reasoning in the proof of Theorem 
$11,|h|^{2}=|p|^{2} w_{0}$ where $p$ has $n$ zeros on $\mathbf{T}$ which contradicts the strong outer property of $h$. Thus $\operatorname{dim} M_{1}=\infty$, and $\operatorname{dim} M_{k+1} \geq \operatorname{dim}\left(M_{1}\right)-k$ $=\infty$ for all $k \geq 1$. This, together with [2, Lemma 4.6], provides evidence for the following conjecture: if $S_{\phi}$ contains more than one function one of which is strong outer, then $S_{\phi}$ contains a function with an inner factor which is not a finite Blaschke product. Note that it is easy to construct examples of processes for which $\mathscr{P} \cap \mathscr{F}_{k} \neq\{0\}$ for all $k>1$. The spectral density $w=|1+B|^{2}$ is such an example if $B$ is an inner function which is not a finite Blaschke product.

The authors would like to thank the referee for pointing out an error in an earlier version of the paper.

\section{REFERENCES}

1. V. M. Adamian, D. Z. Arov and M. G. Krein, On infinite Hankel matrices and generalized problems of Caratheodory-Fejer and F. Riesz, Funkcional. Anal. i Prilozen., 2 (1968) vyp. 1, 1-19.

2. K. deLeeuw and W. Rudin, Extreme points and extremum problems in $H^{1}$, Pacific J. Math., 8 (1958), 467-485.

3. J. B. Garnett, Two remarks on interpolation by bounded analytic functions, in Banach spaces of analytic functions, Lecture Notes in Math. no. 604, Springer-Verlag, Berlin (1977), 32-40.

4. H. Helson and D. E. Sarason, Past and future, Math. Scand., 21 (1967), 5-16.

5. K. Hoffman, Banach Spaces of Analytic Functions, Prentice-Hall, Englewood Cliffs, N. J., 1962.

6. A. N. Kolmogorov, Stationary sequences in Hilbert space, Bull. Moscow State Univ., 2 No. 6 (1941), 1-40.

7. M. G. Krein, On an extrapolation problem of A. N. Kolmogorov, Dokl. Akad. Nauk. SSSR, 46 (1944), 306-309.

8. N. Levinson and H. P. McKean, Jr., Weighted trigonometrical approximation on $\mathbf{R}^{1}$ with application to the germ field of a stationary Gaussian noise, Acta. Math., 112 (1964), 99-143.

9. Y. A. Rozanov, Stationary Random Processes, Holden-Day, San Francisco, 1967.

10. D. E. Sarason, Function Theory on the Unit Circle, Notes for Lectures at a conference at Virginia Polytechnic and State University, Virginia (1978).

11. G. Szego, Beitrage zur Theorie der Toeplitzchen Formen, Math. Z. 6 (1920), 167-202.

Received October 30, 1981.

DePaRTMENT OF Statistics

PRINCETON UNIVERSITY

PRINCETON, NJ 08544

Biomedical and Environmental Health SCiences

UNIVERSITY OF CALIFORNIA

BERKELEY, CA 94720

DePaRTMENT OF MATHEMATICS

FORDHAM UNIVERSITY

NeW YoRK, NY 10458 



\title{
PACIFIC JOURNAL OF MATHEMATICS \\ EDITORS
}

\author{
DONALD BABBITT (Managing Editor) \\ University of California \\ Los Angeles, CA 90024 \\ Hugo Rossi \\ University of Utah \\ Salt Lake City, UT 84112 \\ C. C. Moore and Arthur Ogus \\ University of California \\ Berkeley, CA 94720
}

\author{
J. DugundiI \\ Department of Mathematics \\ University of Southern California \\ Los Angeles, CA 90089-1113
}

R. FINN and H. SAMELSON

Stanford University

Stanford, CA 94305

\begin{tabular}{lllll}
\multicolumn{3}{c}{ ASSOCIATE EDITORS } \\
R. ARENS & $\begin{array}{lll}\text { E. F. BeCKendach } \\
(1906-1982)\end{array}$ & B. H. NeumanN & F. Wolf & K. Yoshida
\end{tabular}

\begin{tabular}{ll}
\multicolumn{2}{c}{ SUPPORTING INSTITUTIONS } \\
UNIVERSITY OF ARIZONA & UNIVERSITY OF OREGON \\
UNIVERSITY OF BRITISH COLUMBIA & UNIVERSITY OF SOUTHERN CALIFORNIA \\
CALIFORNIA INSTITUTE OF TECHNOLOGY & STANFORD UNIVERSITY \\
UNIVERSITY OF CALIFORNIA & UNIVERSITY OF HAWAII \\
MONTANA STATE UNIVERSITY & UNIVERSITY OF TOKYO \\
UNIVERSITY OF NEVADA, RENO & UNIVERSITY OF UTAH \\
NEW MEXICO STATE UNIVERSITY & WASHINGTON STATE UNIVERSITY \\
OREGON STATE UNIVERSITY & UNIVERSITY OF WASHINGTON
\end{tabular}

The Supporting Institutions listed above contribute to the cost of publication of this Journal, but they are not owners or publishers and have no responsibility for its content or policies.

Mathematical papers intended for publication in the Pacific Journal of Mathematics should be in typed form or offset-reproduced (not dittoed), double spaced with large margins. Please do not use built up fractions in the text of the manuscript. However, you may use them in the displayed equations. Underline Greek letters in red, German in green, and script in blue. The first paragraph must be capable of being used separately as a synopsis of the entire paper. In particular it should contain no bibliographic references. Please propose a heading for the odd numbered pages of less than 35 characters. Manuscripts, in triplicate, may be sent to any one of the editors. Please classify according to the scheme of Math. Reviews, Index to Vol. 39. Supply name and address of author to whom proofs should be sent. All other communications should be addressed to the managing editor, or Elaine Barth, University of California, Los Angeles, California 90024.

There are page-charges associated with articles appearing in the Pacific Journal of Mathematics. These charges are expected to be paid by the author's University, Government Agency or Company. If the author or authors do not have access to such Institutional support these charges are waived. Single authors will receive 50 free reprints; joint authors will receive a total of 100 free reprints. Additional copies may be obtained at cost in multiples of 50 .

The Pacific Journal of Mathematics is issued monthly as of January 1966. Regular subscription rate: $\$ 132.00$ a year (6 Vol., 12 issues). Special rate: $\$ 66.00$ a year to individual members of supporting institutions.

Subscriptions, orders for numbers issued in the last three calendar years, and changes of address should be sent to Pacific Journal of Mathematics, P.O. Box 969, Carmel Valley, CA 93924, U.S.A. Old back numbers obtainable from Kraus Periodicals Co., Route 100, Millwood, NY 10546.

The Pacific Journal of Mathematics ISSN 0030-8730 is published monthly by the Pacific Journal of Mathematics at P.O. Box 969, Carmel Valley, CA 93924. Application to mail at Second-class postage rates is pending at Carmel Valley, California, and additional mailing offices. Postmaster: Send address changes to Pacific Journal of Mathematics, P. O. Box 969, Carmel Valley, CA 93924.

PUBLISHED BY PACIFIC JOURNAL OF MATHEMATICS, A NON-PROFIT CORPORATION

Copyright $\odot 1983$ by Pacific Journal of Mathematics 


\section{Pacific Journal of Mathematics}

Vol. 107, No. $2 \quad$ February, 1983

Driss Abouabdillah, Topologies de corps $A$ linéaires $\ldots \ldots \ldots \ldots \ldots . \ldots 257$

Patrick Robert Ahern, On the behavior near a torus of functions

holomorphic in the ball $\ldots \ldots \ldots \ldots \ldots \ldots \ldots \ldots \ldots \ldots \ldots \ldots \ldots . \ldots 267$

Donald Werner Anderson, There are no phantom cohomology operations

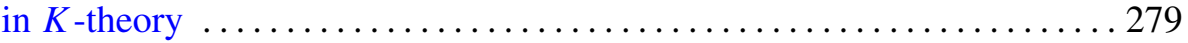

Peter Bloomfield, Nicolas P. Jewell and Eric Hayashi, Characterizations of

completely nondeterministic stochastic processes . ............. 307

Sydney Dennis Bulman-Fleming and K. McDowell, Absolutely flat

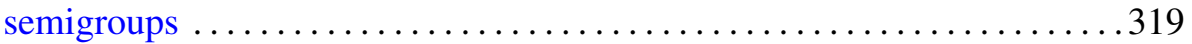

C. Debiève, On a Radon-Nikodým problem for vector-valued measures . . . 335

Dragomir Z. Djokovic, Products of positive reflections in real orthogonal

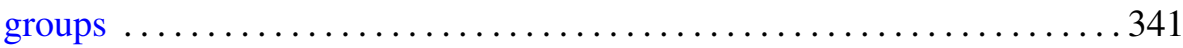

Thomas Farmer, The dual of the nilradical of the parabolic subgroups of

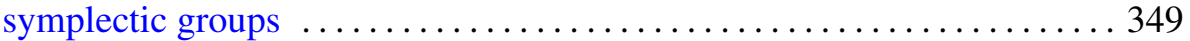

Gary R. Greenfield, Uniform distribution in subgroups of the Brauer group of an algebraic number field $\ldots \ldots \ldots \ldots \ldots \ldots \ldots \ldots \ldots \ldots \ldots . \ldots \ldots$

Paul Daniel Hill, When $\operatorname{Tor}(A, B)$ is a direct sum of cyclic groups $\ldots \ldots \ldots 383$

Hiroshi Maehara, Regular embeddings of a graph $\ldots \ldots \ldots \ldots \ldots \ldots \ldots 3$

Nikolaos S. Papageorgiou, Nonsmooth analysis on partially ordered vector spaces. I. Convex case . .............................4 403

Louis Jackson Ratliff, Jr., Powers of ideals in locally unmixed Noetherian

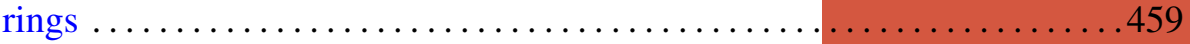

F. Dennis Sentilles and Robert Francis Wheeler, Pettis integration via the Stonian transform .......................... 